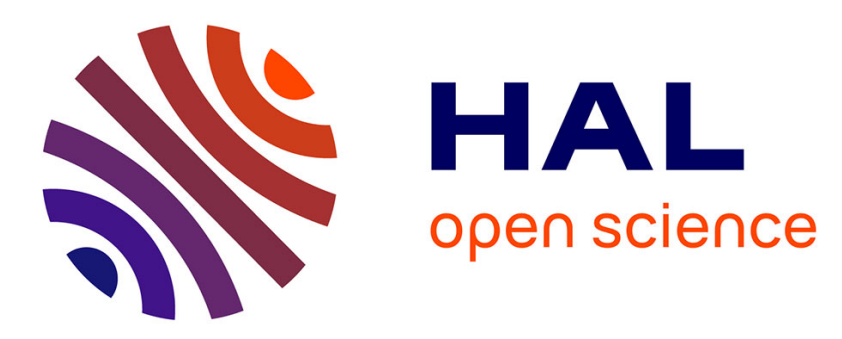

\title{
A new method for solving Pareto eigenvalue complementarity problems
}

Samir Adly, Hadia Rammal

\section{To cite this version:}

Samir Adly, Hadia Rammal. A new method for solving Pareto eigenvalue complementarity problems. Computational Optimization and Applications, 2013, 55 (3), pp.703-731. 10.1007/s10589-013-9534-y . hal-00908170

\section{HAL Id: hal-00908170 \\ https://hal-unilim.archives-ouvertes.fr/hal-00908170}

Submitted on 17 Jul 2018

HAL is a multi-disciplinary open access archive for the deposit and dissemination of scientific research documents, whether they are published or not. The documents may come from teaching and research institutions in France or abroad, or from public or private research centers.
L'archive ouverte pluridisciplinaire HAL, est destinée au dépôt et à la diffusion de documents scientifiques de niveau recherche, publiés ou non, émanant des établissements d'enseignement et de recherche français ou étrangers, des laboratoires publics ou privés. 


\title{
A new method for solving Pareto eigenvalue complementarity problems
}

\author{
Samir Adly • Hadia Rammal
}

\begin{abstract}
In this paper, we introduce a new method, called the Lattice Projection Method (LPM), for solving eigenvalue complementarity problems. The original problem is reformulated to find the roots of a nonsmooth function. A semismooth Newton type method is then applied to approximate the eigenvalues and eigenvectors of the complementarity problems. The LPM is compared to $\mathrm{SNM}_{\min }$ and $\mathrm{SNM}_{\mathrm{FB}}$, two methods widely discussed in the literature for solving nonlinear complementarity problems, by using the performance profiles as a comparing tool (Dolan, Moré in Math. Program. 91:201-213, 2002). The performance measures, used to analyze the three solvers on a set of matrices mostly taken from the Matrix Market (Boisvert et al. in The quality of numerical software: assessment and enhancement, pp. 125137, 1997), are computing time, number of iterations, number of failures and maximum number of solutions found by each solver. The numerical experiments highlight the efficiency of the LPM and show that it is a promising method for solving eigenvalue complementarity problems. Finally, Pareto bi-eigenvalue complementarity problems were solved numerically as an application to confirm the efficiency of our method.
\end{abstract}

Keywords Complementarity problems · One-constrained eigenvalue problems . Complementarity functions · Semismooth Newton Method - Lattice Projection Method $\cdot$ Bi-eigenvalue complementarity problems

Dedicated to Jonathan Borwein in honor of his 60th birthday.

S. Adly $(\bowtie) \cdot H$. Rammal

XLIM UMR-CNRS 7252, Université de Limoges, 87060 Limoges, France

e-mail: samir.adly@unilim.fr

H. Rammal

e-mail: hadia.rammal@xlim.fr 


\section{Introduction}

Since the last three decades, the subject of complementarity problems has become one of the most well-established disciplines within mathematical programming. Due to its various applications in engineering, economics and sciences, it is not surprising that many researchers have focused their attention on developing both theoretical results and efficient numerical methods for solving these problems (see the exhaustive survey [11]). Among these problems, we focus, in our study, on the Eigenvalue Complementarity Problems (EiCP), also called, cone-constrained eigenvalue problems [1, 18-20, 32, 33, 36, 38, 39] that are considered as nonlinear optimization problems. These problems consist to find a scalar (eigenvalue) and a nonzero vector (eigenvector) satisfying a complementarity relation over a closed convex cone in a finite-dimensional space. This case recovers the classical eigenvalue problem when the closed convex cone coincides with the whole space. The Pareto-eigenvalue problems correspond to the case when the closed convex cone coincides with the positive orthant $\mathbb{R}_{+}^{n}$. A wide variety of applications in sciences and engineering require the numerical computation of the solutions of EiCP such as the dynamic analysis of structural mechanical systems, vibro-acoustic systems, electrical circuit simulation, signal processing, fluid dynamic, contact problem in mechanics (see for instance [24-28]). In some applications, the knowledge of eigenvalues can avoid the instability and unwanted resonance for a given system. In mechanical structures for example, eigenvalues are closely related to resonance frequency and to the stability analysis of the corresponding dynamical systems. The computation of eigenvalues becomes crucial in order to be located and damped eigenvalues corresponding to unstable modes or involving large vibrations (see [42]).

The theoretical spectral analysis is now well-developed (see [32]), while the construction of robust and efficient algorithms, for solving EiCP, needs further investigation. Note that the number of Pareto-eigenvalues grows exponentially with the dimension of the problem, for example, a matrix of order 25 may have more than 3 million Pareto-eigenvalues. Therefore, finding the whole Pareto-spectrum of a medium or a large size matrix is not an easy task. Recently, S. Adly and A. Seeger [1] reformulated the EiCP as an equivalent nonsmooth system of equations, by using the Nonlinear Complementarity Functions (NCP-functions) $\phi_{\min }$ and $\phi_{\mathrm{FB}}$, and then applied the corresponding Semismooth Newton Method $\left(\mathrm{SNM}_{\min }\right.$ and $\left.\mathrm{SNM}_{\mathrm{FB}}\right)$. The major contribution of this paper is to introduce a new method, namely the Lattice Projection Method (LPM for short), for solving EiCP. This method is not based on the complementarity approach since it does not use any NCP-functions. The LPM is compared to $\mathrm{SNM}_{\mathrm{min}}$ and $\mathrm{SNM}_{\mathrm{FB}}$ by using the performance profiles as a comparison tool [10] and the numerical experiments highlight that the LPM solver is efficient and robust. We have also studied under which conditions all the elements of the Clarke generalized Jacobians $\partial \Phi\left(z^{*}\right)$ defined in (18) and $\partial \Phi_{\mathrm{LPM}}\left(\tilde{z}^{*}\right)$ defined in (25) at solutions $z^{*}$ and $\tilde{z}^{*}$ of (17) and (24), respectively, are nonsingular.

The structure of this paper is as follows. In the next section, some theoretical results related to EiCP are briefly discussed. In Sect. 3, we establish a short description of the Semismooth Newton Method by presenting the algorithm and its convergence theorem. Moreover, we reformulate the EiCP as an equivalent nonsmooth system 
of equations, by using the Nonlinear Complementarity Functions (NCP-functions) $\phi_{\min }$ and $\phi_{\mathrm{FB}}$. The new method, called Lattice Projection Method (LPM), for solving EiCP is discussed in Sect. 4. In Sect. 5, we study the nonsingularity conditions of the Clarke generalized Jacobians $\partial \Phi\left(z^{*}\right)$ and $\partial \Phi_{\mathrm{LPM}}\left(\tilde{z}^{*}\right)$ defined in (18) and (25), respectively. Some examples are discussed to illustrate this regularity concept. We provide, in Sect. 6, some numerical results related to some matrices of order 3, 4 and 5 (known to have the maximum number of Pareto-eigenvalues). In Sect. 7, we give some extensive numerical tests showing that the LPM is extremely robust and very effective. Performance profiles and data, mostly taken from the Matrix Market [3], are used to analyze the performance of the three algorithms: LPM, SNM min $_{\text {}}$ and $\mathrm{SNM}_{\mathrm{FB}}$. Computing time, number of iterations, number of failures and maximum number of eigenvalues found by each solver are used as performance measures to compare these algorithms. In Sect. 8, we show that the LPM can be generalized to solve the bivariate Eigenvalue Complementarity problems (bi-EiCP) [38]. Numerical results are then reported to confirm the efficiency and the robustness of the LPM. Finally, in Sect. 9, we close up the paper with a conclusion.

\section{Pareto eigenvalue complementarity problems}

For a real matrix $A \in \mathbb{M}_{n}(\mathbb{R})$ of order $n$, the eigenvalue complementarity problem EiCP associated to the Pareto cone $\mathbb{R}_{+}^{n}$ is defined as follows:

$$
\left\{\begin{array}{l}
\text { find } \lambda \in \mathbb{R} \text { and } x \in \mathbb{R}^{n} \backslash\{0\}, \quad \text { such that } \\
\quad x \geq 0, \lambda x-A x \geq 0,\langle x, \lambda x-A x\rangle=0 .
\end{array}\right.
$$

The scalar $\lambda$ and the vector $x$ satisfying system (1) are respectively called a Paretoeigenvalue of $A$ and an associated Pareto-eigenvector. The set of all eigenvalues is called the Pareto-spectrum of $A$, and it is defined by

$$
\sigma(A)=\left\{\lambda \in \mathbb{R}: \exists x \in \mathbb{R}^{n} \backslash\{0\}, 0 \leq x \perp(\lambda x-A x) \geq 0\right\} .
$$

The following result, due to A. Seeger in [38], fully characterized the Pareto-spectrum of (1).

Lemma 1 [38] The scalar $\lambda$ is a Pareto-eigenvalue of $A$ if and only if there exist a nonempty index set $I \subseteq N=\{1, \ldots, n\}$, and a vector $\eta \in \mathbb{R}^{|I|}$ such that

$$
\begin{aligned}
& A_{I} \eta=\lambda \eta, \quad \eta \in \operatorname{int}\left(\mathbb{R}_{+}^{|I|}\right), \\
& \sum_{j \in I} a_{i j} \eta_{j} \leq 0, \quad \forall i \in N \backslash I,
\end{aligned}
$$

where $A_{I} \in \mathbb{M}_{|I|}(\mathbb{R})$ denotes the principal submatrix of $A$ which is obtained by deleting the ith row and the ith column of $A$, whenever $i \notin I$. In this case, the vector $x \in \mathbb{R}^{n}$ given by

$$
x_{i}= \begin{cases}\eta_{i} & \text { if } i \in I, \\ 0 & \text { if } i \in N \backslash I .\end{cases}
$$

is a Pareto-eigenvector of $A$ associated to the eigenvalue $\lambda$. 
Remark 1 This lemma shows that solving the EiCP is equivalent to solve $2^{n}-1$ classical eigenvalue subproblems involving the sub-matrices $A^{I}$ and select only the solutions satisfying the condition (3). This result can be used to find all Pareto-eigenvalues of a matrix $A$ of small order $n$.

The Pareto capacity of the space $\mathbb{M}_{n}(\mathbb{R})$, is defined by

$$
\pi_{n}=\max _{A \in \mathbb{M}_{n}(\mathbb{R})} \operatorname{card}[\sigma(A)],
$$

where $\operatorname{card}[\sigma(A)]$ is the cardinality of the Pareto-spectrum of $A$. As proved in $[32,40]$, one has

$$
3\left(2^{n-1}-1\right) \leq \pi_{n} \leq n 2^{n-1}-(n-1),
$$

which shows that the number of eigenvalues grows exponentially with respect to the dimension $n$. We have $\pi_{1}=1, \pi_{2}=3$ while $\pi_{3}$ is either 9 or 10 . We note also for example that $\pi_{20} \geq 1572861$ and that obtaining an explicit formula for $\pi_{n}$ or improving the lower and the upper bounds in (4) are open questions. The lower bound in (4) is not sharp. In fact, the matrix $A_{2}$ of order 4 (given in Sect. 6) has 23 Paretoeigenvalues, which means that $\pi_{4} \geq 23$, while the lower bound in (4) gives $\pi_{4} \geq 21$.

In many applications, the Pareto-eigenvalue $\lambda$ is assumed to be $>0$ (see for example [18-20]). In the rest of the paper, we will assume that $\lambda>0$. By shifting the matrix $A$, it is possible to find nonpositive Pareto-eigenvalues.

\section{The Semismooth Newton Method SNM}

We start this section by reminding the reader some basic tools from nonsmooth analysis, and some basic properties of the Semismooth Newton Method (SNM). For more details, we refer to $[34,37,43]$ and references therein.

Consider a mapping $\Phi: \mathbb{R}^{n} \rightarrow \mathbb{R}^{n}$ supposed to be locally Lipschitz. The $B$-subdifferential of $\Phi$ at a point $z \in \mathbb{R}^{n}$ is defined by

$$
\partial_{B} \Phi(z)=\left\{M \in \mathbb{M}_{n}(\mathbb{R}): \exists\left(z_{k}\right) \subset D_{\Phi}: z_{k} \rightarrow z \text { and } \lim _{k \rightarrow+\infty} \nabla \Phi\left(z_{k}\right)=M\right\},
$$

where $D_{\Phi}$ is the set of differentiability points of $\Phi$. The Clarke generalized Jacobian [5] of $\Phi$ is given by

$$
\partial \Phi(z)=\operatorname{co}_{B} \Phi(z),
$$

where "co" stands for the convex hull of the set $\partial_{B} \Phi(z)$.

The function $\Phi$ is said to be semismooth $[29,37]$ at $z \in \mathbb{R}^{n}$ if it is locally Lipschitz around $z$, directionally differentiable at $z$ and satisfies the following condition

$$
\sup _{M \in \partial \Phi(z+h)}\|\Phi(z+h)-\Phi(z)-M z\|=o(\|h\|) .
$$

If we replace $o(\|h\|)$ by $O\left(\|h\|^{2}\right)$ in (6), then $\Phi$ is said to be strongly semismooth at $z$. 
We note that semismooth functions are between Lipschitz continuous functions and continuously differentiable functions. For example, the Euclidean norm and piecewise differentiable functions are semismooth. Therefore, the functions $\phi: \mathbb{R}^{2} \rightarrow \mathbb{R},\left(x_{1}, x_{2}\right) \mapsto \phi_{\max }\left(x_{1}, x_{2}\right)=\max \left(x_{1}, x_{2}\right), \phi_{\min }\left(x_{1}, x_{2}\right)=\min \left(x_{1}, x_{2}\right)$ and $\phi_{\mathrm{FB}}\left(x_{1}, x_{2}\right)=x_{1}+x_{2}-\sqrt{x_{1}^{2}+x_{2}^{2}}$ are semismooth.

Given a locally Lipschitz function $\Phi: \mathbb{R}^{n} \rightarrow \mathbb{R}^{n}$, we consider the following nonlinear system of equations

$$
\Phi(x)=0 \mathbb{R}^{n} .
$$

We note that many problems in engineering and sciences can be formulated as (7).

Next, we present the standard algorithm of the Semismooth Newton Method SNM, which will be used later.

\section{Algorithm SNM}

Initialization. Choose an initial point $z^{0}$ and set $k=0$.

Iteration. ${ }^{1}$ One has a current point $z^{k}$. Choose $M^{k} \in \partial \Phi\left(z^{k}\right)$ and compute $h^{k}$ by solving the linear system

$$
M^{k} h^{k}=-\Phi\left(z^{k}\right)
$$

Then, set $z^{k+1}=z^{k}+h^{k}$ and $k=k+1$.

To ensure that the linear system (8) admits a unique solution, the matrix $M^{k}$ must be nonsingular. Furthermore, the following theorem gives some conditions to ensure the convergence of the above algorithm [37].

Theorem 1 Let $\bar{z}$ be a zero of the function $\Phi$. Suppose the following

(i) $\Phi$ is semismooth (resp. strongly semismooth) at $\bar{z}$;

(ii) all matrices in $\partial \Phi(\bar{z})$ are nonsingular.

Then, there exists a neighborhood $V$ of $\bar{z}$ such that the SNM initialized at any $z^{0} \in V$ generates a sequence $\left(z^{k}\right)_{k \in \mathbb{N}}$ that converges superlinearly (resp. quadratically) to $\bar{z}$.

Remark 2 Theoretical convergence results of the semismooth Newton method are ensured under the crucial assumption (ii) that all generalized Jacobian are nonsingular at the solution $\bar{z}$. From a computational point of view, the rate of convergence of the SNM will also depend on the conditioning number of the matrices in $\partial \Phi(z)$ when $z$ is around $\bar{z}$.

As a first step towards a reformulation of the EiCP defined in (1), as a system of equations, we write

$$
x \geq 0, \quad y \geq 0, \quad\langle x, y\rangle=0, \quad \lambda x-A x-y=0, \quad\left\langle\mathbf{1}_{n}, x\right\rangle-1=0,
$$

\footnotetext{
${ }^{1}$ As stopping criteria, we use $\left\|\Phi\left(z^{k}\right)\right\|<10^{-8}$.
} 
where $\mathbf{1}_{n}$ is a vector of ones. The last condition in (9) is used to ensure that $x$ is a nonzero vector. One can also use some other normalization conditions as $\|x\|_{2}^{2}=1$.

Using the NCP-functions, it is possible to write the first three conditions in (9) in the form $U_{\phi}(x, y)=0_{\mathbb{R}^{n}}$ where $U_{\phi}: \mathbb{R}^{n} \times \mathbb{R}^{n} \rightarrow \mathbb{R}^{n}$ is the vector function defined by

$$
U_{\phi}(x, y)=\left[\begin{array}{c}
\phi\left(x_{1}, y_{1}\right) \\
\vdots \\
\phi\left(x_{n}, y_{n}\right)
\end{array}\right],
$$

and $\phi: \mathbb{R}^{2} \rightarrow \mathbb{R}$ is a NCP-function, i.e.

$$
\phi(a, b)=0 \quad \Longleftrightarrow \quad a \geq 0, b \geq 0, a b=0 .
$$

More precisely, we are led to solve the following system of $2 n+1$ equations

$$
\begin{gathered}
U_{\phi}(x, y)=0_{\mathbb{R}^{n}}, \\
\lambda x-A x-y=0 \mathbb{R}^{n}, \\
\left\langle\mathbf{1}_{n}, x\right\rangle-1=0 .
\end{gathered}
$$

In this paper, we focus on the NCP-functions, the most widely used in the literature, namely the Fischer-Burmeister (FB) function [12] and the natural residual function $[14,31]$, also called the $\min$ function, defined respectively by

$$
\begin{gathered}
\phi_{\mathrm{FB}}(a, b)=a+b-\sqrt{a^{2}+b^{2}}, \\
\phi_{\min }(a, b)=\min (a, b) .
\end{gathered}
$$

We note that other NCP-functions $[7,22,35]$ can be treated in the same way.

Consider the vector function $\Phi: \mathbb{R}^{n} \times \mathbb{R}^{n} \times \mathbb{R} \rightarrow \mathbb{R}^{2 n+1}, z=(x, y, \lambda) \longmapsto$ $\Phi(x, y, \lambda)$ defined by

$$
\Phi(z)=\Phi(x, y, \lambda)=\left[\begin{array}{c}
U_{\phi}(x, y) \\
\lambda x-A x-y \\
\left\langle\mathbf{1}_{n}, x\right\rangle-1
\end{array}\right] .
$$

Therefore, the EiCP can be reformulated as a system of nonlinear and nonsmooth equations, i.e. $(x, y, \lambda)$ is a solution of problem (1) if and only if

$$
\Phi(x, y, \lambda)=0_{\mathbb{R}^{2 n+1}} .
$$

Lemma 2 The function $\Phi$ defined in (16) is semismooth with $\phi$ being one of the NCPfunctions $\phi_{\min }$ or $\phi_{\mathrm{FB}}$. Moreover, its Clarke generalized Jacobian at $z=(x, y, \lambda)$ is given by

$$
\partial \Phi(z)=\left\{\left[\begin{array}{ccc}
E & F & 0 \\
\lambda \mathbf{I}_{n}-A & -\mathbf{I}_{n} & x \\
\mathbf{1}_{n}^{T} & 0 & 0
\end{array}\right]:[E, F] \in \partial U_{\phi}(x, y)\right\},
$$

with $\mathbf{I}_{n}$ representing the identity matrix of order $n$. 
$E=\operatorname{diag}\left(a_{1}, \ldots, a_{n}\right), \quad F=\operatorname{diag}\left(b_{1}, \ldots, b_{n}\right) \in \mathbb{M}_{n}(\mathbb{R})$ are diagonal matrices whose ith diagonal element is given by the following two cases:

case 1: if $\phi=\phi_{\mathrm{FB}}$, then, for $i=1, \ldots, n$,

$$
\left\{\begin{array}{l}
a_{i}=1-\frac{x_{i}}{\left\|\left(x_{i}, y_{i}\right)\right\|} \\
b_{i}=1-\frac{y_{i}}{\left\|\left(x_{i}, y_{i}\right)\right\|}
\end{array} \quad \text { if }\left(x_{i}, y_{i}\right) \neq(0,0),\right.
$$

and

$$
\left\{\begin{array}{l}
a_{i}=1-\xi_{i} \\
b_{i}=1-\rho_{i}
\end{array} \quad \text { if }\left(x_{i}, y_{i}\right)=(0,0),\right.
$$

for every $\left(\xi_{i}, \rho_{i}\right) \in \mathbb{R}^{2}$ such that $\left\|\left(\xi_{i}, \rho_{i}\right)\right\| \leq 1$.

case 2: if $\phi=\phi_{\min }$, then, for $i=1, \ldots, n$,

$$
\begin{cases}a_{i}=0, b_{i}=1 & \text { if } x_{i}>y_{i}, \\ a_{i}=1, b_{i}=0 & \text { if } x_{i}<y_{i}, \\ a_{i}=\alpha_{i}, b_{i}=1-\alpha_{i}, & \text { if } x_{i}=y_{i} \text { such that } \alpha_{i} \in[0,1] .\end{cases}
$$

Proof The proof of (18) is given in [1] while the proofs of (19), (20) and (21) can be found in [30].

\section{The Lattice Projection Method (LPM)}

In this section, we introduce a new formulation of the EiCP which leads us to solve a nonlinear and nonsmooth system of $(2 n+1)$ equations involving the same number of variables. The originality of this formulation, in comparison with the existing literature, is that it is not based on the complementarity approach, i.e. we do not use any NCP-functions. We transform the EiCP to find the eigenvalues of a nonlinear problem. The following lemma is in this sense. Without loss of generality, we will assume in the sequel that $\lambda>0$. If the matrix $A$ has a nonpositive eigenvalue $\lambda<0$, we set $\tilde{A}=A+\mu I_{n}$ with $\mu>0$ and large enough. It is easy to see, in this case that the spectrum $\sigma(\tilde{A}) \subset \mathbb{R}_{+}^{*}$.

Lemma 3 The EiCP defined in (1) with $\lambda>0$ is equivalent to find the roots of the following nonlinear and nonsmooth function $f: \mathbb{R}^{n} \times \mathbb{R}_{+}^{*} \rightarrow \mathbb{R}^{n}$ defined by

$$
(x, \lambda) \longmapsto f(x, \lambda)=(A x)^{+}-\lambda x,
$$

where $(A x)^{+}=\max (A x, 0)$ is the positive componentwise.

Proof Suppose $\lambda>0$ and $x \in \mathbb{R}^{n} \backslash\{0\}$. EiCP defined in (1)

$$
0 \leq x \perp(\lambda x-A x) \geq 0
$$


is equivalent to the following problem

$$
\min (x, \lambda x-A x)=0,
$$

where the min is taken componentwise. Since $\lambda>0$, we have the following

$$
\begin{aligned}
\min (x, \lambda x-A x)=0 & \Longleftrightarrow \min (\lambda x, \lambda x-A x)=0 \\
& \Longleftrightarrow \max (-\lambda x, A x-\lambda x)=0 \\
& \Longleftrightarrow \max (A x, 0)=\lambda x,
\end{aligned}
$$

which completes the proof.

Remark 3 Lemma 3 showed that

$$
0 \leq x \perp(\lambda x-A x) \geq 0 \quad \Longleftrightarrow \quad(A x)^{+}=\lambda x,
$$

which means that the EiCP is equivalent to solve the nonlinear eigenvalue problem

$$
\left(\mathbf{P}_{\mathbb{R}_{+}^{n}} \circ A\right)(x)=\lambda x,
$$

where $\mathbf{P}_{\mathbb{R}_{+}^{n}}$ is the projection operator over $\mathbb{R}_{+}^{n}$.

In the rest of this section, we are led to solve the following system of $2 n+1$ equations

$$
\left\{\begin{array}{l}
\tilde{y}^{+}-\lambda x=0 \mathbb{R}^{n}, \\
A x-\tilde{y}=0 \mathbb{R}^{n}, \\
\left\langle\mathbf{1}_{n}, x\right\rangle-1=0 .
\end{array}\right.
$$

We use the normalization condition $\left\langle\mathbf{1}_{n}, x\right\rangle=1$ to ensure that $x$ is a nonzero eigenvector.

Let us consider the following function $\Phi_{\mathrm{LPM}}: \mathbb{R}^{n} \times \mathbb{R}^{n} \times \mathbb{R} \rightarrow \mathbb{R}^{2 n+1}$ defined by

$$
\Phi_{\mathrm{LPM}}(x, \tilde{y}, \lambda)=\left[\begin{array}{c}
\tilde{y}^{+}-\lambda x \\
A x-\tilde{y} \\
\left\langle\mathbf{1}_{n}, x\right\rangle-1
\end{array}\right] .
$$

It is clear that solving EiCP is equivalent to solving the nonlinear system

$$
\Phi_{\mathrm{LPM}}(x, \tilde{y}, \lambda)=0_{\mathbb{R}^{2 n+1}} .
$$

We will use the SNM Algorithm presented in Sect. 3 to solve the generalized equation (24). This method will be called Lattice Projection Method (LPM). In Sects. 6 and 7, the LPM will be compared to $\mathrm{SNM}_{\mathrm{FB}}$ and $\mathrm{SNM}_{\min }$.

The following lemma gives a brief description of the representation of the Clarke generalized Jacobian of $\Phi_{\mathrm{LPM}}$. 
Lemma 4 The function $\Phi_{\mathrm{LPM}}$, defined in (23), is semismooth. Moreover, its Clarke generalized Jacobian at $\tilde{z}=(x, \tilde{y}, \lambda)$ is given by the following block representation

$$
\partial \Phi_{\mathrm{LPM}}(\tilde{z})=\left\{\left[\begin{array}{ccc}
-\lambda \mathbf{I}_{n} & \tilde{F} & -x \\
A & -\mathbf{I}_{n} & 0 \\
\mathbf{1}_{n}^{T} & 0 & 0
\end{array}\right]: \tilde{F} \in \partial(\cdot)^{+}(\tilde{y})\right\},
$$

where $\tilde{F}=\operatorname{diag}\left(u_{1}, \ldots, u_{n}\right) \in \mathbb{R}^{n \times n}$ is a diagonal matrix whose ith diagonal element is given by

$$
u_{i}=\frac{1+\operatorname{Sign}\left(\tilde{y}_{i}\right)}{2}= \begin{cases}1 & \text { if } \tilde{y}_{i}>0 \\ {[0,1]} & \text { if } \tilde{y}_{i}=0 \\ 0 & \text { if } \tilde{y}_{i}<0\end{cases}
$$

Proof Follows immediately by using Theorem (Chain Rule II) in [5].

\section{Nonsingularity conditions}

In this section, we study under which conditions all the elements of the Clarke generalized Jacobians $\partial \Phi\left(z^{*}\right)$ defined in (18) and $\partial \Phi_{\mathrm{LPM}}\left(\tilde{z}^{*}\right)$ defined in (25) at solutions $z^{*}$ and $\tilde{z}^{*}$ of (17) and (24), respectively, are nonsingular.

We begin first by fixing some notations that will be used later.

The index set $\{1,2, \ldots, n\}$ will be abbreviated by the capital letter $I$. If $M=$ $\left(m_{i j}\right) \in \mathbb{M}_{n}(\mathbb{R})$ is a matrix and $\mathcal{J}, \mathcal{K} \subseteq I$, then $M_{\mathcal{J K}}$ denotes the submatrix in $\mathbb{M}_{|\mathcal{J}|,|\mathcal{K}|}(\mathbb{R})$ with elements $m_{i j}, i \in \mathcal{J}, j \in \mathcal{K}$.

We recall that a matrix $M \in \mathbb{M}_{n}(\mathbb{R})$ is a P-matrix if all its principal minors are strictly positive. Furthermore, $M$ is a P-matrix if and only if for every nonzero vector $x \in \mathbb{R}^{n} \backslash\{0\}$, there exist an index $i \in I$ such that $x_{i} \neq 0$ and $x_{i}[M x]_{i}>0$.

Let us now introduce the function $F: \mathbb{R}^{n} \times \mathbb{R}^{n} \times \mathbb{R} \rightarrow \mathbb{R}^{2 n+1}$ defined by

$$
z=(x, y, \lambda) \longmapsto \quad F(z)=\left[\begin{array}{c}
y \\
\lambda x-A x-y \\
\left\langle\mathbf{1}_{n}, x\right\rangle-1
\end{array}\right] .
$$

It is easy to see that $F$ is of class $C^{1}$.

Proposition 1 Any $H \in \partial \Phi(z)$, with $\phi$ being one of the NCP-functions $\phi_{\min }$ or $\phi_{\mathrm{FB}}$, can be written in the form

$$
H=D_{a}+D_{b} F^{\prime}(z),
$$

where $D_{a}, D_{b} \in \mathbb{M}_{2 n+1}(\mathbb{R})$ are two positive semidefinite diagonal matrices defined by

$$
D_{a}=\operatorname{diag}\left(a_{i}\right)_{1 \leq i \leq 2 n+1} \quad \text { and } \quad D_{b}=\operatorname{diag}\left(b_{i}\right)_{1 \leq i \leq 2 n+1},
$$

whose ith diagonal element being one of the two cases mentioned above in (19), (20) and (21) for all $i=1, \ldots, n$ and $a_{i}=0, b_{i}=1$ for $i=n+1, \ldots, 2 n+1$. 
Proof Let $H \in \partial \Phi(z)$. By Lemma 2, we have

$$
H=\left[\begin{array}{ccc}
E & F & 0 \\
\lambda \mathbf{I}_{n}-A & -\mathbf{I}_{n} & x \\
\mathbf{1}_{n}^{T} & 0 & 0
\end{array}\right] .
$$

On the other hand, we have

$$
F^{\prime}(z)=\left[\begin{array}{ccc}
0 & \mathbf{I}_{n} & 0 \\
\lambda \mathbf{I}_{n}-A & -\mathbf{I}_{n} & x \\
\mathbf{1}_{n}^{T} & 0 & 0
\end{array}\right]
$$

Hence, from these equalities and using [21, Proposition 2.6], the statement of the proposition follows easily and we get

$$
H=D_{a}+D_{b} F^{\prime}(z)
$$

To deal with the nonsingularity of all Clarke generalized Jacobian in $\partial \Phi\left(z^{*}\right)$ at a solution $z^{*}=\left(x^{*}, y^{*}, \lambda^{*}\right)$, let us introduce some notations.

The function $G: \mathbb{R}^{n} \times \mathbb{R} \rightarrow \mathbb{R}^{n+1}$ is defined by

$$
w=(x, \lambda) \quad \longmapsto \quad G(w)=\left[\begin{array}{c}
\lambda x-A x \\
\left\langle\mathbf{1}_{n}, x\right\rangle-1
\end{array}\right] .
$$

We set

$$
w^{*}=\left(x^{*}, \lambda^{*}\right)
$$

and

$$
J=\Delta_{a}+\Delta_{b} G^{\prime}\left(w^{*}\right),
$$

where $\Delta_{a}=\operatorname{diag}\left(a_{1}, \ldots, a_{n}, 0_{\mathbb{R}}\right), \Delta_{b}=\operatorname{diag}\left(b_{1}, \ldots, b_{n}, 1\right)$ two diagonal matrices of $\mathbb{M}_{n+1}(\mathbb{R})$ with $\left(x_{i}^{*}, y_{i}^{*}\right)=\left(x_{i}^{*},\left(\lambda^{*} x^{*}-A x^{*}\right)_{i}\right)$ at a solution $z^{*}=\left(x^{*}, y^{*}, \lambda^{*}\right)$ and $G^{\prime}\left(w^{*}\right)$ is given by

$$
G^{\prime}\left(w^{*}\right)=\left[\begin{array}{cc}
\lambda \mathbf{I}_{n}^{*}-A & x^{*} \\
\mathbf{1}_{n}^{T} & 0
\end{array}\right]
$$

We note that

$$
\lambda^{*} \Delta_{a}=\Delta_{\lambda^{*} a}
$$

where

$$
\lambda^{*} a=\left(\lambda^{*} a_{1}, \lambda^{*} a_{2}, \ldots, \lambda^{*} a_{n}\right)
$$

We set

$$
\tilde{J}=\Delta_{\lambda * a}+\Delta_{b} G^{\prime}\left(w^{*}\right) .
$$

The following standard index sets will be used:

$$
\alpha:=\left\{i \in\{1, \ldots, n, n+1\}: w_{i}^{*}>0=G_{i}\left(w^{*}\right)\right\},
$$




$$
\begin{aligned}
& \beta:=\left\{i \in\{1, \ldots, n, n+1\}: w_{i}^{*}=0=G_{i}\left(w^{*}\right)\right\}, \\
& \gamma:=\left\{i \in\{1, \ldots, n, n+1\}: w_{i}^{*}=0<G_{i}\left(w^{*}\right)\right\} .
\end{aligned}
$$

Proposition 2 Let $z^{*}=\left(x^{*}, y^{*}, \lambda^{*}\right)$ be a fixed root of $\Phi$ defined in (16) with $w^{*}=$ $\left(x^{*}, \lambda^{*}\right)$. If the submatrix $G^{\prime}\left(w^{*}\right)_{\alpha \alpha}$ is nonsingular and its Schur-complement

$$
G^{\prime}\left(w^{*}\right)_{\beta \beta}-G^{\prime}\left(w^{*}\right)_{\beta \alpha} G^{\prime}\left(w^{*}\right)_{\alpha \alpha}^{-1} G^{\prime}\left(w^{*}\right)_{\alpha \beta}
$$

is a P-matrix, then the Jacobians $J$ and $\tilde{J}$ defined respectively in (30) and (32) are nonsingular matrices.

Proof It is easy to see that the EiCP is equivalent to the following system

$$
\left\{\begin{array}{r}
\text { find } w \in \mathbb{R}^{n+1} \backslash\{0\}, \quad \text { such that } \\
w \geq 0, G(w) \geq 0, \quad\langle w, G(w)\rangle=0,
\end{array}\right.
$$

where $G$ is given by (29).

The desired result follows immediately from Lemma 5.3 in [7] or Theorem 2.8 in [21].

Theorem 2 Assume that $z^{*}=\left(x^{*}, y^{*}, \lambda^{*}\right)$ is a fixed root of $\Phi$ and set $w^{*}=\left(x^{*}, \lambda^{*}\right)$. Then the Jacobian $J$ defined in (30) is nonsingular if and only if all elements $H$ in $\partial \Phi\left(z^{*}\right)$ are nonsingular.

Proof Let $H \in \partial \Phi\left(z^{*}\right)$ and let $X=(p, q, r) \in \mathbb{R}^{n} \times \mathbb{R}^{n} \times \mathbb{R}$ be an arbitrary vector such that

$$
H X=0_{\mathbb{R}^{2 n+1}} .
$$

The previous equation (34) can be written explicitly as

$$
\left[\begin{array}{ccc}
E & F & 0 \\
\lambda^{*} \mathbf{I}_{n}-A & -\mathbf{I}_{n} & x^{*} \\
\mathbf{1}_{n}^{T} & 0 & 0
\end{array}\right]\left[\begin{array}{c}
p \\
q \\
r
\end{array}\right]=\left[\begin{array}{c}
0_{\mathbb{R}^{n}} \\
0_{\mathbb{R}^{n}} \\
0_{\mathbb{R}}
\end{array}\right],
$$

where $E$ and $F$ are defined in Lemma 2. Therefore,

$$
\begin{gathered}
E p+F q=0_{\mathbb{R}^{n}}, \\
\left(\lambda^{*} \mathbf{I}_{n}-A\right) p-q+r x^{*}=0_{\mathbb{R}^{n}}, \\
\left\langle\mathbf{1}_{n}, p\right\rangle=0 .
\end{gathered}
$$

Using (36), we have

$$
q=\left(\lambda^{*} \mathbf{I}_{n}-A\right) p+r x^{*}
$$


Consequently, (35) becomes

$$
E p+F\left(\lambda^{*} \mathbf{I}_{n}-A\right) p+r F x^{*}=0_{\mathbb{R}^{n}} .
$$

Therefore,

$$
\left\{\begin{array}{l}
E p+F\left(\lambda^{*} \mathbf{I}_{n}-A\right) p+r F x^{*}=0 \mathbb{R}^{n}, \\
\left\langle\mathbf{1}_{n}, p\right\rangle=0,
\end{array}\right.
$$

which can be rewritten in the following matricial form

$$
\left[\begin{array}{cc}
E+F\left(\lambda^{*} \mathbf{I}_{n}-A\right) & F x^{*} \\
\mathbf{1}_{n}^{T} & 0
\end{array}\right]\left[\begin{array}{l}
p \\
r
\end{array}\right]=\left[\begin{array}{c}
0_{\mathbb{R}^{n}} \\
0_{\mathbb{R}}
\end{array}\right] .
$$

By setting $Y=(p, r) \in \mathbb{R}^{n} \times \mathbb{R}$, it is clear that (40) is equivalent to

$$
J Y=0_{\mathbb{R}^{n+1}} .
$$

Hence,

$$
H X=0_{\mathbb{R}^{2 n+1}} \Longleftrightarrow\left\{\begin{array}{l}
J Y=0_{\mathbb{R}^{n+1}}, \\
q=\left(\lambda^{*} \mathbf{I}_{n}-A\right) p+r x^{*} .
\end{array}\right.
$$

The desired conclusion of Theorem 2 follows immediately from the last equivalence.

The following theorem gives nonsingularity conditions of the generalized Jacobian of $\Phi_{\mathrm{LPM}}$.

Theorem 3 Assume that $\tilde{z}^{*}=\left(x^{*}, \tilde{y}^{*}, \lambda^{*}\right)$ is a fixed root of $\Phi_{\mathrm{LPM}}$ defined in (23) and set $w^{*}=\left(x^{*}, \lambda^{*}\right)$. Then the Jacobian $\tilde{J}$ defined in (32) is nonsingular if and only if all elements in $\partial \Phi_{\mathrm{LPM}}\left(\tilde{z}^{*}\right)$ are nonsingular.

Proof Let $\tilde{H} \in \partial \Phi_{\mathrm{LPM}}\left(\tilde{z}^{*}\right)$ and let $X=(p, q, r) \in \mathbb{R}^{n} \times \mathbb{R}^{n} \times \mathbb{R}$ be an arbitrary vector such that

$$
\tilde{H} X=0_{\mathbb{R}^{2 n+1}} .
$$

The previous equation (42) can be written explicitly as

$$
\left[\begin{array}{ccc}
-\lambda^{*} \mathbf{I}_{n} & \tilde{F} & -x^{*} \\
A & -\mathbf{I}_{n} & 0 \\
\mathbf{1}_{n}^{T} & 0 & 0
\end{array}\right]\left[\begin{array}{c}
p \\
q \\
r
\end{array}\right]=\left[\begin{array}{c}
0_{\mathbb{R}^{n}} \\
0_{\mathbb{R}^{n}} \\
0_{\mathbb{R}}
\end{array}\right]
$$

where $\tilde{F}$ is defined in Lemma 4. Therefore,

$$
\begin{gathered}
-\lambda^{*} p+\tilde{F} q-r x^{*}=0 \mathbb{R}^{n}, \\
A p-q=0_{\mathbb{R}^{n}} \\
\left\langle\mathbf{1}_{n}, p\right\rangle=0 .
\end{gathered}
$$


Using (44), we have

$$
q=A p
$$

Consequently, (43) becomes

$$
\left(\lambda^{*} \mathbf{I}_{n}-\tilde{F} A\right) p+r x^{*}=0 \mathbb{R}^{n} .
$$

Therefore,

$$
\left\{\begin{array}{l}
\left(\lambda^{*} \mathbf{I}_{n}-\tilde{F} A\right) p+r x^{*}=0 \mathbb{R}^{n}, \\
\left\langle\mathbf{1}_{n}, p\right\rangle=0 .
\end{array}\right.
$$

Notice that $E+F=\mathbf{I}_{n}$ where $E=\operatorname{diag}\left(a_{1}, \ldots, a_{n}\right), F=\operatorname{diag}\left(b_{1}, \ldots, b_{n}\right) \in \mathbb{M}_{n}(\mathbb{R})$ are diagonal matrices whose $i$ th diagonal element is given by (21) and $\phi_{\min }$ being the min function defined in (15). Therefore, (48) becomes

$$
\left\{\begin{array}{l}
\lambda^{*} E p+\left(\lambda^{*} F-\tilde{F} A\right) p+r x^{*}=0 \mathbb{R}^{n}, \\
\left\langle\mathbf{1}_{n}, p\right\rangle=0
\end{array}\right.
$$

Since $y^{*}=\lambda^{*} x^{*}-A x^{*}$, then we get $\tilde{y}^{*}=A x^{*}=\lambda^{*} x^{*}-y^{*}$. Hence, $\tilde{F}=$ $\operatorname{diag}\left(u_{1}, \ldots, u_{n}\right)$ whose $i$ th diagonal element is given by

$$
u_{i}= \begin{cases}1 & \text { if } \lambda^{*} x_{i}^{*}>y_{i}^{*}, \\ {[0,1]} & \text { if } \lambda^{*} x_{i}^{*}=y_{i}^{*}, \\ 0 & \text { if } \lambda^{*} x_{i}^{*}<y_{i}^{*} .\end{cases}
$$

Recall that $F=\operatorname{diag}\left(b_{1}, \ldots, b_{n}\right)$ is defined by

$$
b_{i}= \begin{cases}1 & \text { if } x_{i}^{*}>y_{i}^{*}, \\ {[0,1]} & \text { if } x_{i}^{*}=y_{i}^{*}, \\ 0 & \text { if } x_{i}^{*}<y_{i}^{*} .\end{cases}
$$

Using the complementarity approach

$$
0 \leq x^{*} \perp y^{*} \geq 0
$$

and the fact that $\lambda^{*}>0$, we get

$$
0 \leq \lambda^{*} x^{*} \perp y^{*} \geq 0 \quad \text { and } \quad x_{i}^{*}=0 \text { or } y_{i}^{*}=0 .
$$

Therefore,

$$
\tilde{F}=F \text {. }
$$

We note also that we have obviously

$$
F x^{*}=x^{*} \quad\left(\text { since } E+F=\mathbf{I}_{n}\right) \text {. }
$$


Consequently, (49) becomes

$$
\left\{\begin{array}{l}
\lambda^{*} E p+F\left(\lambda^{*} \mathbf{I}_{n}-A\right) p+r x^{*}=0 \mathbb{R}^{n}, \\
\left\langle\mathbf{1}_{n}, p\right\rangle=0,
\end{array}\right.
$$

which can be rewritten in the following matricial form

$$
\left[\begin{array}{cc}
\tilde{E}+F\left(\lambda^{*} \mathbf{I}_{n}-A\right) & F x^{*} \\
\mathbf{1}_{n}^{T} & 0
\end{array}\right]\left[\begin{array}{l}
p \\
r
\end{array}\right]=\left[\begin{array}{c}
0_{\mathbb{R}^{n}} \\
0_{\mathbb{R}}
\end{array}\right],
$$

where $\tilde{E}=\lambda^{*} E$. By setting $Y=(p, r) \in \mathbb{R}^{n} \times \mathbb{R}$, it is clear that (53) is equivalent to

$$
\tilde{J} Y=0_{\mathbb{R}^{n+1}} .
$$

Hence,

$$
\tilde{H} X=0_{\mathbb{R}^{2 n+1}} \Longleftrightarrow\left\{\begin{array}{l}
\tilde{J} Y=0_{\mathbb{R}^{n+1}}, \\
q=A p .
\end{array}\right.
$$

The desired conclusion of Theorem 3 follows immediately from the last equivalence.

In order to illustrate the above results, we consider the following examples.

Example 1 Consider the following matrix

$$
A=\left[\begin{array}{cc}
8 & -1 \\
3 & 4
\end{array}\right]
$$

Using Lemma 1 , it is easy to see that $\lambda^{*}=4$ is a solution of the EiCP with $x^{*}=$ $(0,1)^{T}$ as an associated eigenvector. We set

$$
z^{*}=(0,1,1,0,4)^{T}, \quad \tilde{z}^{*}=(0,1,-1,4,4),
$$

and we show that $z^{*}$ (respectively $\tilde{z}^{*}$ ) is a "good solution" of the nonlinear equation $\Phi(z)=0$ defined in (17) (respectively $\Phi_{\mathrm{LPM}}(\tilde{z})=0$ defined in (24)).

A direct application of Proposition 2 yields

$$
\begin{aligned}
& G^{\prime}\left(w^{*}\right)=\left[\begin{array}{ccc}
-4 & 1 & 0 \\
-3 & 0 & 1 \\
1 & 1 & 0
\end{array}\right] \text { and } \\
& G^{\prime}\left(w^{*}\right)_{\alpha \alpha}=\left[\begin{array}{ll}
0 & 1 \\
1 & 0
\end{array}\right] \quad \text { (which is a nonsingular matrix), }
\end{aligned}
$$

with $w^{*}=(0,1,4)^{T}, \alpha=\{2,3\}, \beta=\emptyset$ and $\gamma=\{1\}$.

Hence, the first condition of Proposition 2 is satisfied and so is the second, since $\beta$ is empty. Consequently, $J$ and $\tilde{J}$ are nonsingular matrices and so are $H$ and $\tilde{H}$ (by Theorems 2 and 3). 
On the other hand, a direct calculation of the Jacobian matrix $H \in \partial \Phi\left(z^{*}\right)$ shows that it is nonsingular and is given by

$$
H=\left[\begin{array}{ccccc}
1 & 0 & 0 & 0 & 0 \\
0 & 0 & 0 & 1 & 0 \\
-4 & 1 & -1 & 0 & 0 \\
-3 & 0 & 0 & -1 & 1 \\
1 & 1 & 0 & 0 & 0
\end{array}\right],
$$

with $\phi$ being one of the NCP-functions $\phi_{\min }$ or $\phi_{\mathrm{FB}}$.

Moreover, we have

$$
\tilde{F}=F=\left[\begin{array}{ll}
0 & 0 \\
0 & 1
\end{array}\right],
$$

where $\tilde{y}^{*}=A x^{*}=(-1,4)^{T}$. A direct calculation of the Jacobian matrix $\tilde{H} \in$ $\partial \Phi_{\mathrm{LPM}}\left(\tilde{z}^{*}\right)$ shows that it is nonsingular and is given by

$$
\tilde{H}=\left[\begin{array}{ccccc}
-4 & 0 & 0 & 0 & 0 \\
0 & -4 & 0 & 1 & -1 \\
8 & -1 & -1 & 0 & 0 \\
3 & 4 & 0 & -1 & 0 \\
1 & 1 & 0 & 0 & 0
\end{array}\right]
$$

Example 2 Let

$$
B=\left[\begin{array}{ccc}
15 & -2 & 0 \\
-11 & 9 & -1 \\
6 & -10 & 23
\end{array}\right]
$$

Lemma 1 ensures that $\lambda^{*}=23$ is a solution of EiCP associated to $B$ with $x^{*}=$ $(0,0,1)^{T}$ as an eigenvector. In this case, $y^{*}=\lambda^{*} x^{*}-B x^{*}=(0,1,0)^{T}$ and $\tilde{y}^{*}=$ $B x^{*}=(0,-1,23)^{T}$. We set

$$
z^{*}=(0,0,1,0,1,0,23)^{T}, \quad \tilde{z}^{*}=(0,0,1,0,-1,23,23)^{T},
$$

and we show that $z^{*}$ (respectively $\tilde{z}^{*}$ ) is a "good solution" of the nonlinear equation $\Phi(z)=0$ (respectively $\Phi_{\mathrm{LPM}}(\tilde{z})=0$ ). A direct application of Proposition 2 yields

$$
\begin{aligned}
G^{\prime}\left(w^{*}\right) & =\left[\begin{array}{cccc}
8 & 2 & 0 & 0 \\
11 & 14 & 1 & 0 \\
-6 & 10 & 0 & 1 \\
1 & 1 & 1 & 0
\end{array}\right] \text { and } \\
G^{\prime}\left(w^{*}\right)_{\alpha \alpha} & =\left[\begin{array}{ll}
0 & 1 \\
1 & 0
\end{array}\right] \text { (which is a nonsingular matrix), }
\end{aligned}
$$

with $w^{*}=(0,0,1,23)^{T}, \alpha=\{3,4\}, \beta=\{1\}$ and $\gamma=\{2\}$. Moreover, the Schurcomplement of $G^{\prime}\left(w^{*}\right)_{\alpha \alpha}$,

$$
G^{\prime}\left(w^{*}\right)_{\beta \beta}-G^{\prime}\left(w^{*}\right)_{\beta \alpha} G^{\prime}\left(w^{*}\right)_{\alpha \alpha}^{-1} G^{\prime}\left(w^{*}\right)_{\alpha \beta}=8-\left[\begin{array}{ll}
0 & 0
\end{array}\right]\left[\begin{array}{ll}
0 & 1 \\
1 & 0
\end{array}\right]\left[\begin{array}{c}
-6 \\
1
\end{array}\right]=8>0,
$$


is obviously a P-matrix. Hence, $J$ and $\tilde{J}$ are nonsingular and so are $H$ and $\tilde{H}$ by Theorems 2 and 3. On the other hand, a direct calculation of the Jacobian matrix $H$, with $\phi_{\min }$ being the min function defined in (15), yields

$$
H=\left[\begin{array}{ccccccc}
\delta & 0 & 0 & 1-\delta & 0 & 0 & 0 \\
0 & 1 & 0 & 0 & 0 & 0 & 0 \\
0 & 0 & 0 & 0 & 0 & 1 & 0 \\
8 & 2 & 0 & -1 & 0 & 0 & 0 \\
11 & 14 & 1 & 0 & -1 & 0 & 0 \\
-6 & 10 & 0 & 0 & 0 & -1 & 1 \\
1 & 1 & 1 & 0 & 0 & 0 & 0
\end{array}\right],
$$

where $\delta \in[0,1]$. The determinant of $H$ is given by

$$
\operatorname{det}(H)=8-7 \delta .
$$

We note that $H$ is nonsingular since $\delta \in[0,1]$.

Similarly, the determinant of the Jacobian matrix $\tilde{H}$

$$
\tilde{H}=\left[\begin{array}{ccccccc}
-23 & 0 & 0 & \delta & 0 & 0 & 0 \\
0 & -23 & 0 & 0 & 0 & 0 & 0 \\
0 & 0 & -23 & 0 & 0 & 1 & -1 \\
15 & -2 & 0 & -1 & 0 & 0 & 0 \\
-11 & 9 & -1 & 0 & -1 & 0 & 0 \\
6 & -10 & 23 & 0 & 0 & -1 & 0 \\
1 & 1 & 1 & 0 & 0 & 0 & 0
\end{array}\right],
$$

is given by

$$
\operatorname{det}(\tilde{H})=-23(23-15 \delta) .
$$

Hence, $\tilde{H}$ is nonsingular since $\delta \in[0,1]$.

Example 3 Let

$$
C=\left[\begin{array}{cc}
1 & -1 \\
0 & 1
\end{array}\right] .
$$

Analogously to the two examples above, using Lemma $1, \lambda^{*}=1$ is a solution of EiCP associated to $C$ with $x^{*}=(1,0)^{T}$ as an eigenvector, $y^{*}=\lambda^{*} x^{*}-C x^{*}=(0,0)^{T}$ and $\tilde{y}^{*}=C x^{*}=(1,0)^{T}$. We show that

$$
z^{*}=(1,0,0,0,1)^{T} \text { and } \quad \tilde{z}^{*}=(1,0,1,0,1)^{T}
$$

are "bad solutions". A direct calculation of $H \in \partial \Phi\left(z^{*}\right)$ shows that it is not always nonsingular. If we take the NCP-function $\phi_{\min }$ defined in (15), we get

$$
H=\left[\begin{array}{ccccc}
0 & 0 & 1 & 0 & 0 \\
0 & \delta & 0 & 1-\delta & 0 \\
0 & 1 & -1 & 0 & 1 \\
0 & 0 & 0 & -1 & 0 \\
1 & 1 & 0 & 0 & 0
\end{array}\right],
$$


where $\delta \in[0,1]$. We have

$$
\operatorname{det}(H)=-\delta,
$$

which means that $H$ is nonsingular if and only if $\delta \in] 0,1]$.

In the same way, since

$$
\operatorname{det}(\tilde{H})=1-\delta
$$

where $\delta \in[0,1]$, and

$$
\tilde{H}=\left[\begin{array}{ccccc}
-1 & 0 & 1 & 0 & 1 \\
0 & -1 & 0 & \delta & 0 \\
1 & -1 & -1 & 0 & 0 \\
0 & 1 & 0 & -1 & 0 \\
1 & 1 & 0 & 0 & 0
\end{array}\right]
$$

then the Jacobian matrix $\tilde{H}$ is nonsingular if and only if $\delta \in[0,1[$.

On the other hand, using Proposition 2, we get

$$
\begin{aligned}
& G^{\prime}\left(w^{*}\right)=\left[\begin{array}{lll}
0 & 1 & 1 \\
0 & 0 & 0 \\
1 & 1 & 0
\end{array}\right] \text { and } \\
& G^{\prime}\left(w^{*}\right)_{\alpha \alpha}=\left[\begin{array}{ll}
0 & 1 \\
1 & 0
\end{array}\right] \quad \text { (which is a nonsingular matrix), }
\end{aligned}
$$

with $\alpha=\{1,3\}, \beta=\{2\}$ and $\gamma=\emptyset$. However, since the Schur-complement of $G_{\alpha \alpha}^{\prime}\left(w^{*}\right)$ is equal to zero, it is not a P-matrix. Then, the second condition of Proposition 2 is not satisfied.

\section{Testing on matrices of order 3, 4 and 5}

The first numerical experiment will be given by taking matrices of order 3,4 and 5 that are known to have 9, 23 and 57 Pareto-eigenvalues, respectively.

$$
A_{1}=\left[\begin{array}{ccc}
5 & -8 & 2 \\
-4 & 9 & 1 \\
-6 & -1 & 13
\end{array}\right], \quad A_{2}=\left[\begin{array}{cccc}
132 & -106 & 18 & 81 \\
-92 & 74 & 24 & 101 \\
-2 & -44 & 195 & 7 \\
-21 & -38 & 0 & 230
\end{array}\right]
$$

and

$$
A_{3}=\left[\begin{array}{ccccc}
788 & -780 & -256 & 156 & 191 \\
-548 & 862 & -190 & 112 & 143 \\
-456 & -548 & 1308 & 110 & 119 \\
-292 & -374 & -14 & 1402 & 28 \\
-304 & -402 & -66 & 38 & 1522
\end{array}\right]
$$


Table 1 Comparison of the three solvers on the matrices $A_{1}, A_{2}$ and $A_{3}$

\begin{tabular}{|c|c|c|c|c|c|c|c|c|c|}
\hline \multirow[t]{2}{*}{ Methods } & \multicolumn{3}{|l|}{$A_{1}$} & \multicolumn{3}{|l|}{$A_{2}$} & \multicolumn{3}{|l|}{$A_{3}$} \\
\hline & Iter & Time & Failure (\%) & Iter & Time & Failure (\%) & Iter & Time & Failure (\%) \\
\hline LPM & 4 & 0.0003 & 0 & 6 & 0.0006 & 0 & 7 & 0.0004 & 0 \\
\hline $\mathrm{SNM}_{\mathrm{FB}}$ & 8 & 0.0012 & 5 & 10 & 0.0015 & 16 & 11 & 0.0014 & 31 \\
\hline $\mathrm{SNM}_{\text {min }}$ & 2 & 0.0003 & 47 & 2 & 0.0008 & 71 & 2 & 0.0007 & 95 \\
\hline
\end{tabular}

We compare LPM, SNM $\mathrm{FB}$ and $\mathrm{SNM}_{\min }$ by computing the number of iterations, the CPU-time and the number of failures.

Remark 4 In our case, a failure is declared if the Jacobian matrix is ill-conditioned or in the SNM, the number of iterations exceeded 100.

The comparison results are summarized in Table 1 where "Iter" denotes the average iterative number,"Time" denotes the average CPU-time and "Failure (\%)" represents the percentage of the number of failures to find a solution for (EiCP).

We have used $10^{5}$ random initial points, in order to obtain the 57 Paretoeigenvalues of $A_{3}$ simultaneously by the three solvers. We note that LPM finds all eigenvalues of $A_{3}$ by using only $10^{3}$ random initial points.

Conclusion As a first numerical experiment, it is clear that the LPM captures our attention by comparing the percentage of failures of each solver since it has the least percentage. We can also observe that the number of iterations of the $\mathrm{SNM}_{\mathrm{FB}}$ is high compared to the two others.

In the next section, we confirm our result by enlarging the set of tested examples. Moreover, we will compare the CPU time of the three solvers, since for the moment, LPM and $\mathrm{SNM}_{\min }$ have almost the same computing time.

\section{Numerical results}

In this section, we compare the three solvers that have been defined in Sects. 3 and 4, namely $\mathrm{LPM}, \mathrm{SNM}_{\mathrm{FB}}$ and $\mathrm{SNM}_{\min }$. In order, to complete this experience, we choose the performance profiles developed by E.D. Dolan and J.J. Moré [6, 10] as a tool for comparing the solvers. The performance profiles give, for each $t \in \mathbb{R}$, the proportion $\rho_{s}(t)$ of test problems on which each solver under comparison has a performance within the factor $t$ of the best possible ratio.

Computing time, number of iterations, number of failures and maximum number of eigenvalues found by each solver are used as performance measures to compare these algorithms.

Due to the absence of library dedicated to EiCP, we have chosen a set $P$ of 100 matrices, most of them are taken from the Matrix Market [3], with size less than 350. 
Fig. 1 Performance profiles where $t_{p, s}$ is the average computing time (Color figure online)

Fig. 2 Performance profiles where $t_{p, s}$ is the maximum number of solutions (Color figure online)
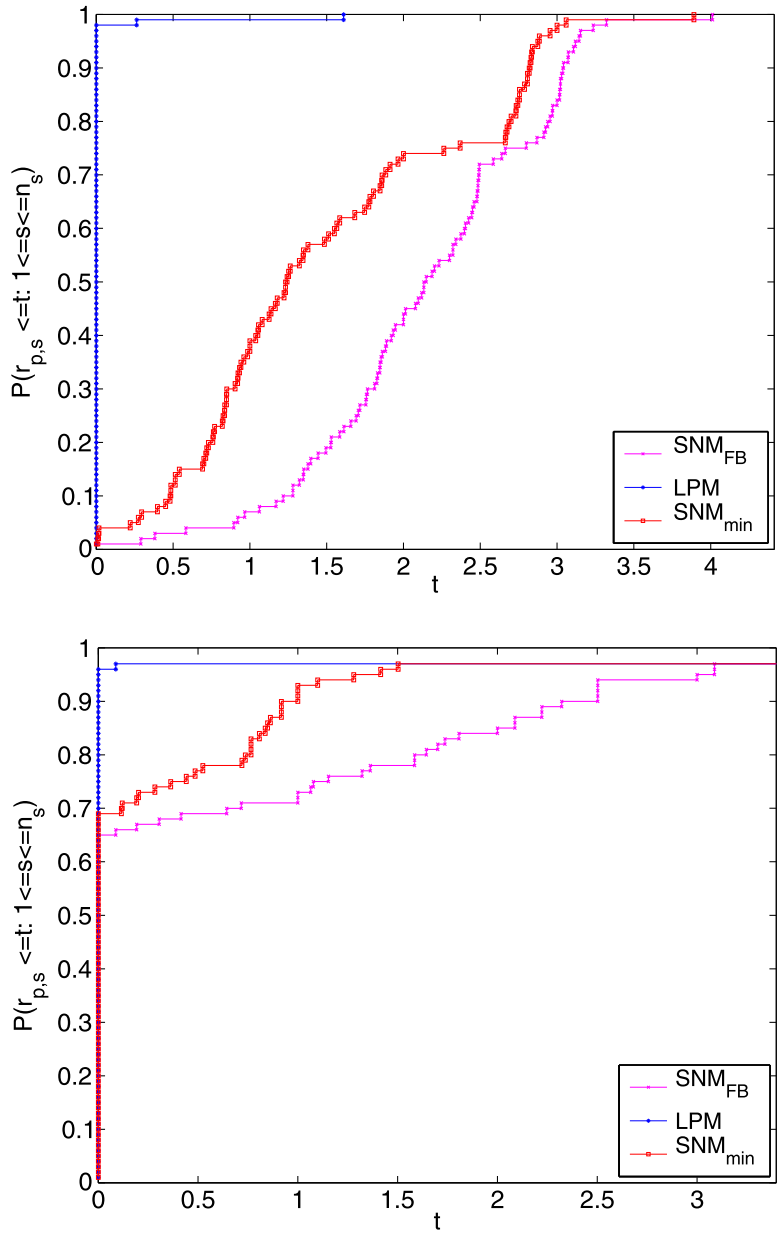

Let $S$ be the set of the three solvers that will be compared and $n_{s}$ the number of solvers. The performance ratio is defined by

$$
r_{p, s}=\frac{t_{p, s}}{\min \left\{t_{p, s}: s \in S\right\}},
$$

where $p \in P, s \in S$, and $t_{p, s}$ is either

- the average CPU time required to solve problem $p$ by solver $s$ corresponding to Fig. 1, or

- the maximum number of solutions corresponding to Fig. 2, or

- the number of failures (in the sense of Remark 4) corresponding to Fig. 3, or

- the average iterative number corresponding to Fig. 4.

The performance of the solver $s \in S$ is defined by

$$
\rho_{s}(t)=\frac{1}{n_{p}} \operatorname{size}\left\{p \in P: r_{p, s} \leq t\right\},
$$


Fig. 3 Performance profiles where $t_{p, s}$ is the number of failures (Color figure online)
Fig. 4 Performance profiles where $t_{p, s}$ is the average iterative number (Color figure online)
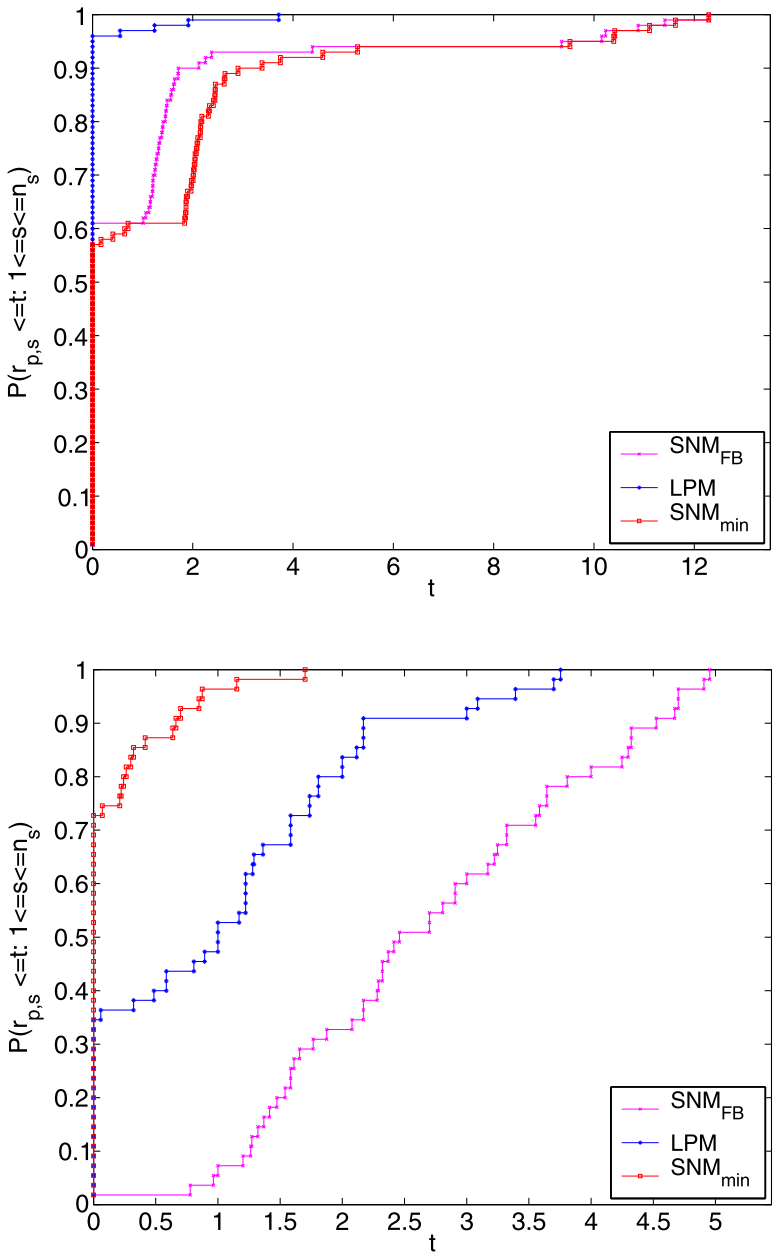

where, $n_{p}$ is the number of problems, and $t$ is a real factor. In this case, $\rho_{s}(t)$ is the probability, for solver $s \in S$, that the performance ratio $r_{p, s}$ is within a factor $t \in \mathbb{R}$ of the best possible ratio. For more details, we refer to [10].

Thanks to the performance profile plot, one can compare how well a solver can perform relative to the other by taking in consideration the four criteria listed above. The value of $\rho_{s}(1)$ gives the probability that the solver $s$ will win over the two others, while $\rho_{s}(t)$, for large value of $t$, measures the robustness of the solver $s$.

The numerical experiments are carried out in a Powerbook Mac OS 10.6.8 with a processor $2.33 \mathrm{GHz}$ Intel Core 2 Duo and 2 Go memory. All the program codes are written and executed in Matlab 7.7.

Figures 1, 2, 3, and 4 show the performance profiles of each solver and a detailed description of the tested problems is given.

Figure 1 presents the performance profiles of the three solvers corresponding to the average computing time. We observe that the LPM is efficient and robust. In fact, in the interval [0, 1], LPM can solve $99 \%$ of the problems, while $\mathrm{SNM}_{\mathrm{FB}}$ and $\mathrm{SNM}_{\min }$ 
do not reach $40 \%$ and require a long running time. $\mathrm{SNM}_{\mathrm{FB}}$ has a lower number of wins than either LPM and $\mathrm{SNM}_{\min }$. However, for $t \geq 2$, the three solvers confirm their robustness.

Figure 1 also indicates that relative to the computing time, with the same initial points and under the same stopping criterion, LPM is the fastest solver, followed respectively by $\mathrm{SNM}_{\min }$ and $\mathrm{SNM}_{\mathrm{FB}}$.

In Fig. 2, the performance ratio represents the maximum number of solutions found by each solver. We remark that the LPM is the winner, it is able to solve $99 \%$ of the problems and detects the maximum number of Pareto-eigenvalues. $\mathrm{SNM}_{\mathrm{FB}}$ has a lower number of wins than either LPM or $\mathrm{SNM}_{\min }$ but its performance increases with the growth of the factor $t$.

The LPM captures our attention in Fig. 3, with its ability to solve over $99 \%$ of problems. Performance ratio represents here the number of failures to solve each problem by each solver (see Remark 4). Another point of interest is that the number of failures giving by $\mathrm{SNM}_{\min }$ is important than the other solvers. We conclude again that the LPM is the winner followed respectively by $\mathrm{SNM}_{\mathrm{FB}}$ and $\mathrm{SNM}_{\min }$.

In Fig. 4, performance ratio displays the average iterative number for each solver. It is clear that $\mathrm{SNM}_{\min }$ has the most wins, followed by LPM and $\mathrm{SNM}_{\mathrm{FB}}$. We observe that the performance of LPM becomes interesting when $t \geq 2$.

We conclude that LPM confirms again its efficiency, robustness and its ability to solve EiCP. By comparing the three solvers on a set of problems with respect to the computing time, number of iterations, number of failures and maximum number of found eigenvalues, LPM performs better than the two other solvers.

\section{Applications to Pareto bivariate Eigenvalue Complementarity Problems (bi-EiCP)}

We define, in this section, the so-called, bivariate Eigenvalue Complementarity Problems (bi-EiCP for short), in the case of Pareto cones. Surprisingly, the numerical simulations for this subject have never been studied. We propose to generalize the LPM, defined in Sect. 4, and to use the same tools mentioned in the previous section as performance measures to compare our method with $\mathrm{SNM}_{\mathrm{FB}}$ and $\mathrm{SNM}_{\min }$ for solving bi-EiCP.

\subsection{The classical bi-Eigenvalue Problems (bi-EiP)}

Let $A, B, C$ and $D$ be real matrices of order $n \times n, n \times m, m \times n$ and $m \times m$, respectively. The classical unconstrained bivariate Eigenvalue Problem is defined by

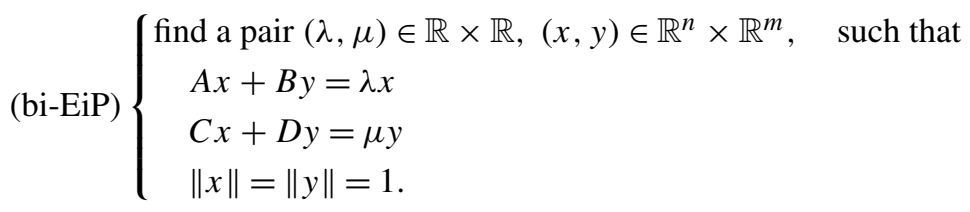


The problem (55) can be rewritten in the equivalent form

$$
\left\{\begin{array}{l}
E z=\Lambda z \\
\left\|z_{i}\right\|=1, \quad i=1,2
\end{array}\right.
$$

where

$$
E=\left[\begin{array}{ll}
A & B \\
C & D
\end{array}\right], \quad \Lambda:=\operatorname{diag}\left\{\lambda \mathbf{I}_{n}, \mu \mathbf{I}_{m}\right\} \quad \text { and } \quad z=\left[\begin{array}{l}
z_{1} \\
z_{2}
\end{array}\right]=\left[\begin{array}{l}
x \\
y
\end{array}\right] .
$$

Bi-EiP arises in many fields of applied mathematics and engineering [23, 41]. They are necessary for the understanding of the constrained bi-EiCP. Bi-EiP was first studied by Hotelling in a brief paper [16] in 1935. His definitive study of the problem appeared one year later in [17] and still stands as a key reference in multivariate statistical literature. In 1961, an iterative method, proposed by Horst [15], has been used for solving bi-EiP. Three decades later, Chu and Watterson [4] proved the convergence of Horst's iterative method. Among recent contributions of bi-EiP, we can cite [13] and references therein. In this section, we propose the use of SNM to solve bi-EiCP.

\subsection{Pareto bi-Eigenvalue Complementarity Problems}

For real matrices $A \in \mathbb{M}_{n}(\mathbb{R}), B \in \mathbb{M}_{n, m}(\mathbb{R}), C \in \mathbb{M}_{m, n}(\mathbb{R})$ and $D \in \mathbb{M}_{m}(\mathbb{R})$, the bi-EiCP associated to the Pareto cones $\left(\mathbb{R}_{+}^{n}, \mathbb{R}_{+}^{m}\right)$ is written as follows:

$$
\left\{\begin{array}{c}
\text { find }(\lambda, \mu) \in \mathbb{R} \times \mathbb{R} \text { and }(x, y) \in \mathbb{R}^{n} \backslash\{0\} \times \mathbb{R}^{m} \backslash\{0\}, \quad \text { such that } \\
x \geq 0, \lambda x-A x-B y \geq 0,\langle x, \lambda x-A x-B y\rangle=0, \\
y \geq 0, \mu y-C x-D y \geq 0,\langle y, \mu y-C x-D y\rangle=0 .
\end{array}\right.
$$

The couple $(\lambda, \mu) \in \mathbb{R} \times \mathbb{R}$ is called the Pareto-bi-eigenvalue of the block structured matrix $E$.

In [2], A. Amri and A. Seeger generalize Lemma 1 to solve bi-EiCP. They characterized the Pareto-bi-eigenvalue (57). The following Lemma is in this sense.

Lemma 5 [2] Given a pair $(\lambda, \mu) \in \mathbb{R} \times \mathbb{R}$, the following statements are equivalent:

(i) Problem (57) has a nonzero solution $(x, y) \in \mathbb{R}^{n} \times \mathbb{R}^{m}$.

(ii) There are index sets $I \subseteq N=\{1, \ldots, n\}$ and $J \subseteq M=\{1, \ldots, m\}$ such that

$$
\left\{\begin{array}{l}
A_{I I} \xi+B_{I J} \eta=\lambda \xi \\
C_{J I} \xi+D_{J J} \eta=\mu \eta
\end{array}\right.
$$

has a solution $(\xi, \eta) \in \mathbb{R}^{|I|+|J|}$ satisfying the interiority conditions

$$
\xi \in \operatorname{int}\left(\mathbb{R}_{+}^{|I|}\right), \quad \eta \in \operatorname{int}\left(\mathbb{R}_{+}^{|J|}\right)
$$


and the binding conditions

$$
\begin{aligned}
& \sum_{k \in I} a_{i k} \xi_{k}+\sum_{l \in J} b_{i l} \eta_{l} \leq 0, \quad \forall i \in N \backslash I, \\
& \sum_{k \in I} c_{j k} \xi_{k}+\sum_{l \in J} d_{j l} \eta_{l} \leq 0, \quad \forall j \in M \backslash J .
\end{aligned}
$$

Furthermore, $(x, y)$ can be constructed from $(\xi, \eta)$ by setting

$$
x_{i}=\left\{\begin{array}{ll}
\xi_{i} & \text { if } i \in I, \\
0 & \text { if } i \in N \backslash I,
\end{array} \quad \text { and } \quad y_{j}= \begin{cases}\eta_{j} & \text { if } j \in J, \\
0 & \text { if } j \in M \backslash J\end{cases}\right.
$$

Without loss of generality, we will assume that $\lambda, \mu>0$. In the same manner as EiCP, as a first step towards a reformulation of bi-EiCP as a system of equations, we write

$$
\begin{array}{rlrl}
x & \geq 0, \quad y \geq 0, \\
u & \geq 0, \quad v \geq 0, \\
u^{T} x & =0, \quad v^{T} y=0, \\
\lambda x-A x-B y-u & =0, & \mu y-C x-D y-v=0, \\
\left\langle\mathbf{1}_{n}, x\right\rangle-1 & =0, & \left\langle\mathbf{1}_{m}, y\right\rangle-1=0 .
\end{array}
$$

Using NCP-functions, the first three conditions above can be rewritten in the succinct form $\mathcal{U}_{\varphi}(x, u)=0$ and $\mathcal{V}_{\varphi}(y, v)=0$, where $\mathcal{U}_{\varphi}: \mathbb{R}^{2 n} \rightarrow \mathbb{R}^{n}$ and $\mathcal{V}_{\varphi}: \mathbb{R}^{2 m} \rightarrow \mathbb{R}^{m}$ are respectively given by

$$
\mathcal{U}_{\varphi}(x, u)=\left[\begin{array}{c}
\varphi\left(x_{1}, u_{1}\right) \\
\vdots \\
\varphi\left(x_{n}, u_{n}\right)
\end{array}\right], \quad \mathcal{V}_{\varphi}(y, v)=\left[\begin{array}{c}
\varphi\left(y_{1}, v_{1}\right) \\
\vdots \\
\varphi\left(y_{m}, v_{m}\right)
\end{array}\right] .
$$

Here, $\varphi$ is one of the NCP-functions (14) or (15).

In short, we are led to solve the following system of $d=2(n+m+1)$ equations

$$
\Phi(z)=0_{\mathbb{R}^{d}},
$$

where $\Phi: \mathbb{R}^{d} \rightarrow \mathbb{R}^{d}$ is a locally Lipschitz function defined by

$$
\Phi(z)=\Phi(x, u, y, v, \lambda, \mu)=\left[\begin{array}{c}
\mathcal{U}_{\varphi}(x, u) \\
\mathcal{V}_{\varphi}(y, v) \\
\lambda x-A x-B y-u \\
\mu y-C x-D y-v \\
\left\langle\mathbf{1}_{n}, x\right\rangle-1 \\
\left\langle\mathbf{1}_{m}, y\right\rangle-1
\end{array}\right] .
$$


Lemma 6 The Clarke generalized Jacobian of $\Phi$ at $z=(x, u, y, v, \lambda, \mu)$ is given by

$$
\partial \Phi(z)=\left[\begin{array}{cccccc}
E_{1} & F_{1} & 0 & 0 & 0 & 0 \\
0 & 0 & E_{2} & F_{2} & 0 & 0 \\
\lambda \mathbf{I}_{n}-A & -\mathbf{I}_{n} & -B & 0 & x & 0 \\
-C & 0 & \mu \mathbf{I}_{m}-D & -\mathbf{I}_{m} & 0 & y \\
\mathbf{1}_{n}^{T} & 0 & 0 & 0 & 0 & 0 \\
0 & 0 & \mathbf{1}_{m}^{T} & 0 & 0 & 0
\end{array}\right],
$$

with $\left[E_{1}, F_{1}\right] \in \partial \mathcal{U}_{\varphi}(x, u)$ and $\left[E_{2}, F_{2}\right] \in \partial \mathcal{V}_{\varphi}(y, v)$, where $E_{1}, F_{1}, E_{2}$ and $F_{2}$ can be computed in the same way as in Lemma 2.

\subsection{Lattice Projection Method applied to bi-EiCP}

In this section, we reformulate the bi-EiCP into a system of nonlinear equations. The following lemma is in this sense.

Lemma 7 Solving bi-EiCP is equivalent to find the roots of the following nonlinear and semismooth function $\Phi_{\mathrm{LPM}}: \mathbb{R}^{d} \rightarrow \mathbb{R}^{d}$ defined by

$$
\Phi_{\mathrm{LPM}}(z)=\Phi_{\mathrm{LPM}}(x, u, y, v, \lambda, \mu)=\left[\begin{array}{c}
u^{+}-\lambda x \\
A x+B y-u \\
v^{+}-\mu y \\
C x+D y-v \\
\left\langle\mathbf{1}_{n}, x\right\rangle-1 \\
\left\langle\mathbf{1}_{n}, y\right\rangle-1
\end{array}\right] .
$$

The Clarke generalized Jacobian of $\Phi_{\mathrm{LPM}}$ at $z=(x, u, y, v, \lambda, \mu)$ is given by

$$
\partial \Phi_{\mathrm{LPM}}(z)=\left[\begin{array}{cccccc}
-\lambda \mathbf{I}_{n} & E_{3} & 0 & 0 & -x & 0 \\
A & -\mathbf{I}_{n} & B & 0 & 0 & 0 \\
0 & 0 & -\mu \mathbf{I}_{m} & F_{3} & 0 & -y \\
C & 0 & D & -\mathbf{I}_{m} & 0 & 0 \\
\mathbf{1}_{n}^{T} & 0 & 0 & 0 & 0 & 0 \\
0 & \mathbf{1}_{m}^{T} & 0 & 0 & 0 & 0
\end{array}\right],
$$

where $E_{3} \in \partial(\cdot)^{+}(u)$ and $F_{3} \in \partial(\cdot)^{+}(v)$ (see Lemma 4).

\subsection{Numerical experiments for solving bi-EiCP}

Similarly to Sect. 7, we study here the following three solvers:

(i) $\mathrm{SNM}_{\mathrm{FB}}$, where $\Phi: \mathbb{R}^{d} \rightarrow \mathbb{R}^{d}$ is defined in (63) and $\varphi$ is the Fischer-Burmeister function (14),

(ii) $\mathrm{SNM}_{\min }$, where $\Phi$ is defined in (63) and $\varphi$ is the min-function (15),

(iii) The generalization of the LPM, where $\Phi_{\text {LPM }}$ is defined in (65). 
Fig. 5 Performance profiles where $t_{p, s}$ is the average computing time (Color figure online)

Fig. 6 Performance profiles where $t_{p, s}$ is the number of failures (Color figure online)
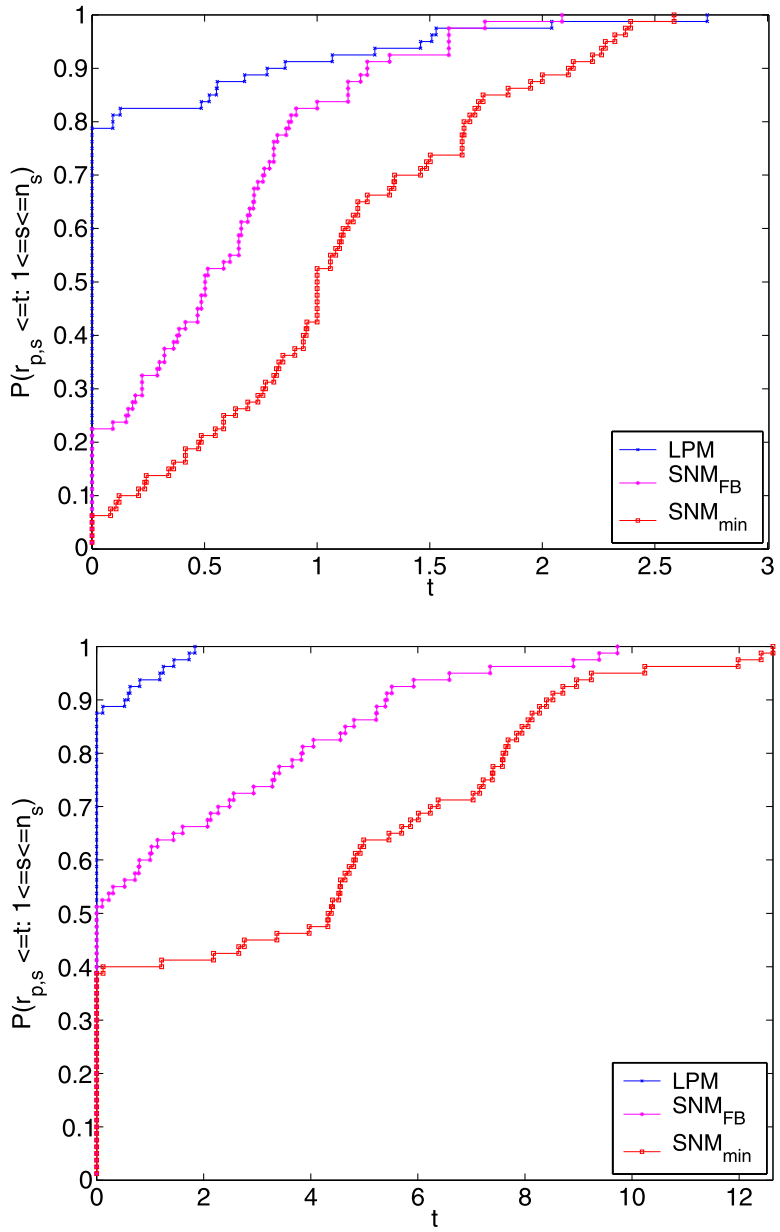

For the comparison, we may use computing time, number of failures, maximum number of solutions and number of iterations as performance measures. Due to the long time required for the simultaneous execution of solvers, we restrict our analysis to a set of 80 matrices for solving bi-EiCP.

In Fig. 5, LPM captures our attention since it has the most wins and it does not require a long running time to solve bi-EiCP. Relative to the computing time, LPM is the fastest solver, followed respectively by $\mathrm{SNM}_{\mathrm{FB}}$ and $\mathrm{SNM}_{\min }$.

Figure 6 represents the performance profiles of the three solvers with respect to the number of failures. We observe that LPM is able to solve $99 \%$ of problems, whereas $\mathrm{SNM}_{\min }$ has a lower number of wins.

In Fig. 7, LPM confirms its ability to detect the maximum number of solutions. We also note that the performance of $\mathrm{SNM}_{\min }$ is lower.

In Fig. 8, we compare the number of iterations corresponding to the common set of eigenvalues of each problem for the three solvers. With respect to this criterion, the performance of LPM and $\mathrm{SNM}_{\min }$ are very close, while $\mathrm{SNM}_{\mathrm{FB}}$ has a lower number of wins. We note that when $t \geq 0.5$, the three solvers are comparable. 
Fig. 7 Performance profiles where $t_{p, s}$ is the maximum number of solutions (Color figure online)

Fig. 8 Performance profiles where $t_{p, s}$ is the average iterative number (Color figure online)

Fig. 9 Performance profiles of $A^{+}$and $\mathrm{SNM}_{\mathrm{FB}}$ where $t_{p, s}$ is the average iterative number (Color figure online)
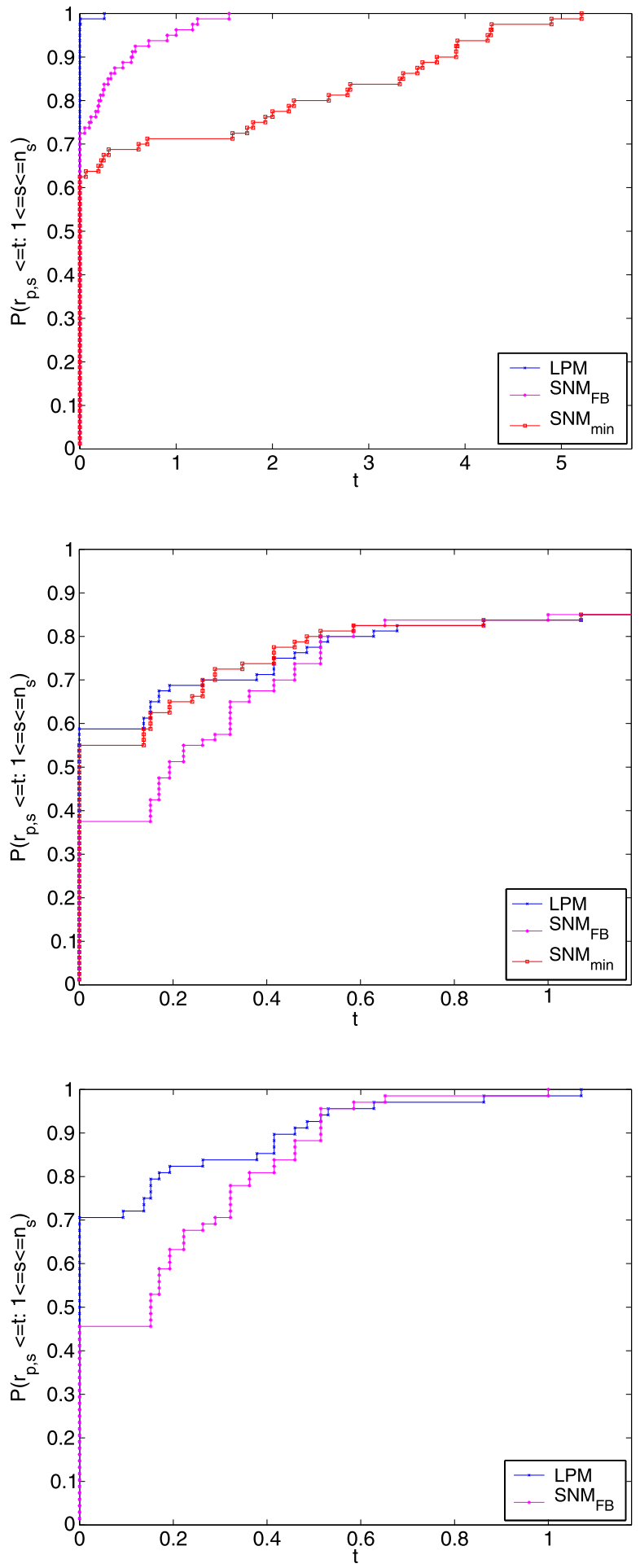
Due to the difference of the performance between (LPM, $\mathrm{SNM}_{\mathrm{FB}}$ ) and $\mathrm{SNM}_{\min }$, with respect to the maximum number of solutions (see Fig. 7), the performance given in Fig. 8 for the three solvers does not reach $86 \%$.

In Fig. 9, we represent only the performance profiles of LPM and $\mathrm{SNM}_{\mathrm{FB}}$ corresponding to the average iterative number. We note that both solvers solve $95 \%$ of problems.

It is clear that, in this figure, the number of iterations of $\mathrm{SNM}_{\mathrm{FB}}$ is more important than LPM. SNM $\mathrm{FB}$ needs more iterations to solve each problem. LPM has the most wins, the robustness of the two solvers is confirmed when $t \geq 0.6$.

\section{Concluding remarks}

In this paper, we proposed a new algorithm, namely the Lattice Projection Method (LPM), for solving Eigenvalue Complementarity Problems and bi-Eigenvalue Complementarity Problems. This method is compared to $\mathrm{SNM}_{\min }$ and $\mathrm{SNM}_{\mathrm{FB}}$, two algorithms widely discussed in the literature for solving nonlinear complementarity problems. The performance profiles assessment, evaluated on more than 100 benchmark datasets, indicate the efficiency and the robustness of the LPM. It would be interesting to consider the globalization of the LPM and compare it to other solvers like PATH-solver $[8,9]$. This is out of the scope of this paper and will probably be the subject of a future project of research. Another forthcoming work will involve the extension of the proposed algorithm to the second order cone (also called the Lorentz cone).

Acknowledgements The first author wishes to thank Professor Jonathan Borwein for some stimulating discussions about the new method LPM and particularly Lemma 3.

The authors wish to thank also Professor Alberto Seeger for his careful reading of the first version of the manuscript.

\section{References}

1. Adly, S., Seeger, A.: A nonsmooth algorithm for cone-constrained eigenvalue problems. Comput. Optim. Appl. 49, 299-318 (2011)

2. Amri, A., Seeger, A.: Spectral analysis of coupled linear complementarity problems. Linear Algebra Appl. 432, 2507-2523 (2010)

3. Boisvert, R.F., Pozo, R., Remington, K., Barrett, R.F., Dongarra, J.J.: Matrix market: a web resource for test matrix collections. In: The Quality of Numerical Software: Assessment and Enhancement, pp. 125-137. Chapman and Hall, London (1997)

4. Chu, M.T., Watterson, J.L.: On a multivariate eigenvalue problem: I. Algebric theory and a power method. SIAM J. Sci. Comput. 14, 1089-1106 (1993)

5. Clarke, F.H.: Optimization and Nonsmooth Analysis. Wiley, New York (1983). Reprinted by, SIAM, Philadelphia, PA, 1990

6. Cops: http://mcs.anl.gov/ more/cops/

7. De Luca, T., Facchinei, F., Kanzow, C.: A semismooth equation approach to the solution of nonlinear complementarity problems. Math. Program. 75, 407-439 (1996)

8. Dirkse, S.P., Ferris, M.C.: A pathsearch damped Newton method for computing general equilibria. Ann. Oper. Res. 68, 211-232 (1996)

9. Dirkse, S.P., Ferris, M.C.: The path solver: a non-monotone stabilization scheme for mixed complementarity problems. Optim. Methods Softw. 5, 123-156 (1995) 
10. Dolan, E.D., Moré, J.J.: Benchmarking optimization software with performance profiles. Math. Program. 91, 201-213 (2002)

11. Ferris, M.C., Pang, J.S.: Engineering and economic applications of complementarity problems. J. Soc. Ind. Appl. Math. 39, 669-713 (1997)

12. Fischer, A.: A special Newton-type optimization method. Optimization 24, 269-284 (1992)

13. Hanafi, M., Ten Berge, J.M.F.: Global optimality of the successive Maxbet algorithm. Psychometrika 68, 97-103 (2003)

14. Harker, P.T., Pang, J.S.: Finite-dimensional variational inequality and nonlinear complementarity problem: a survey of theory, algorithms and applications. Math. Program. 48, 161-220 (1990)

15. Horst, P.: Relations among $m$ sets of measures. Psychometrika 26, 129-149 (1961)

16. Hotelling, H.: The most predictable criterion. J. Educ. Psychol. 26, 139-142 (1935)

17. Hotelling, H.: Relations between two sets of variates. Biometrika 28, 321-377 (1936)

18. Judice, J.J., Sherali, H.D., Ribeiro, I.M.: The eigenvalue complementarity problem. Comput. Appl. 37(2), 139-156 (2007)

19. Júdice, J.J., Raydan, M., Rosa, S.S., Santos, S.A.: On the solution of the symmetric eigenvalue complementarity problem by the spectral projected gradient algorithm. Numer. Algorithms 47, 391-407 (2008)

20. Júdice, J.J., Sherali, H.D., Ribeiro, I.M., Rosa, S.S.: On the asymmetric eigenvalue complementarity problem. Optim. Methods Softw. 24(4-5), 549-568 (2009)

21. Kanzow, C., Kleinmichel, H.: A new class of semismooth Newton type methods for nonlinear complementarity problems. Comput. Optim. Appl. 11, 227-251 (1998)

22. Kanzow, C., Yamashita, N., Fukushima, M.: New NCP-functions and their properties. J. Optim. Theory Appl. 94, 115-135 (1997)

23. Kettenring, J.R.: Canonical analysis of several sets of variables. Biometrika 58, 433-451 (1971)

24. Martins, J.A.C., Barbarin, S., Raous, M., Pinto da Costa, A.: Dynamic stability of finite dimensional linearly elastic systems with unilateral contact and Coulomb friction. Comput. Methods Appl. Mech. Eng. 177, 289-328 (1999)

25. Martins, J.A.C., Pinto da Costa, A.: Stability of finite-dimensional nonlinear elastic systems with unilateral contact and friction. Int. J. Solids Struct. 37, 2519-2564 (2000)

26. Martins, J.A.C., Pinto da Costa, A.: Bifurcations and instabilities in frictional contact problems: theoretical relations, computational methods and numerical results. In: European Congress on Computational Methods in Applied Sciences and Engineering: ECCOMAS (2004)

27. Martins, J.A.C., Pinto da Costa, A.: Computation of bifurcations and instabilities in some frictional contact problems. In: European Conference on Computational Mechanics: ECCM (2001)

28. Martins, J.A.C., Pinto da Costa, A., Figueiredo, I.N., Júdice, J.J.: The directional instability problem in systems with frictional contacts. Comput. Methods Appl. Mech. Eng. 193, 357-384 (2004)

29. Mifflin, R.: Semismooth and semiconvex functions in constrained optimization. SIAM J. Control Optim. 15, 957-972 (1977)

30. Pang, J.S., Facchinei, F.: Finite-Dimensional Variational Inequalities and Complementarity Problems. Operations Research, vol. 2. Springer, New York (2003)

31. Pang, J.S.: Newton's method for B-differentiable equations. Math. Oper. Res. 15, 311-465 (1990)

32. Pinto da Costa, A., Seeger, A.: Cone-constrained eigenvalue problems: theory and algorithms. Comput. Optim. Appl. 45(1), 25-57 (2008)

33. Pinto da Costa, A., Seeger, A.: Numerical resolution of cone-constrained eigenvalues problems. J. Comput. Appl. Math. 28, 37-61 (2009)

34. Qi, L.: Convergence analysis of some algorithms for solving nonsmooth equations. Math. Oper. Res. 18, 227-244 (1993)

35. Qi, L.: Regular pseudo-smooth NCP and BVIP functions and globally and quadratically convergent generalized Newton methods for complementarity and variational inequality problems. Technical report AMR 97/14, The University of New South Wales, Sydney, Australia (1997)

36. Queiroz, M., Júdice, J.J., Humes, C.: The symmetric eigenvalue complementarity problem. Math. Comput. 73(248), 1849-1863 (2003)

37. Qi, L., Sun, J.: A nonsmooth version of Newton's method. Math. Program. 58, 353-367 (1993)

38. Seeger, A.: Eigenvalue analysis of equilibrium processes defined by linear complementarity conditions. Linear Algebra Appl. 292, 1-14 (1999)

39. Seeger, A., Torki, M.: On eigenvalues induced by a cone constraint. Linear Algebra Appl. 372, 181206 (2003)

40. Seeger, A., Vicente-Pérez, J.: On cardinality of Pareto spectra. Electron. J. Linear Algebra 22, 758766 (2011) 
41. Tanaka, Y.: Some generalized methods of optimal scaling and their asymptotic theories: the case of multiple responses-multiple factors. Ann. Inst. Stat. Math. 30, 329-348 (1978)

42. Tisseur, F., Meerbergen, K.: The quadratic eigenvalue problem. J. Soc. Ind. Appl. Math. 43, 235-286 (2001)

43. Ulbrich, M.: In: Semismooth Newton Methods for Variational Inequalities and Constrained Optimization Problems in Function Spaces. MOS-SIAM Series on Optimization (2011) 